J. Nonlinear Var. Anal. 5 (2021), No. 2, pp. 281-297

Available online at http://jnva.biemdas.com

https://doi.org/10.23952/jnva.5.2021.2.07

\title{
MODIFIED INERTIAL SUBGRADIENT EXTRAGRADIENT ALGORITHMS FOR PSEUDOMONOTONE EQUILIBRIUM PROBLEMS WITH THE CONSTRAINT OF NONEXPANSIVE MAPPINGS
}

\author{
LU-CHUAN CENG \\ Department of Mathematics, Shanghai Normal University, Shanghai 200234, China
}

\begin{abstract}
In this paper, we introduce the modified inertial subgradient extragradient algorithms for solving the pseudomonotone equilibrium problems with the constraint of nonexpansive mappings. In our algorithms, the subgradient projection onto some constructible half-space is used in place of the second minimization problem over a closed convex set, and a new approach for attaining the step-size is provided. Strong convergence theorems are established in the framework of Hilbert spaces.

Keywords. Inertial subgradient extragradient method; Pseudomonotone equilibrium problem; Nonexpansive mapping; Fixed point.
\end{abstract}

\section{INTRODUCTION}

Let $\mathscr{H}$ be a real Hilbert space. Given a nonempty closed convex subset $C \subset \mathscr{H}$ and a sequence $\left\{x_{n}\right\} \subset \mathscr{H}$, we use the notation $x_{n} \rightarrow x$, where $x$ is a point in $\mathscr{H}$, to denote the strong convergence of $\left\{x_{n}\right\}$ to $x$. We use the notation $x_{n} \rightarrow x$, to denote the weak convergence of $\left\{x_{n}\right\}$ to $x$. Given a mapping $S$ defined on $\mathscr{H}$, we denote by $\operatorname{Fix}(S)$ the fixed point set of $S$. Recall that mapping $S$ is said to be $L$-Lipschitz continuous if there exists a positive real number $L$ such that $\|S x-S y\| \leq L\|x-y\|, \forall x, y \in \mathscr{H}$. If $L<1$, then $S$ is called a contractive mapping. If $L=1$, then $S$ is called a nonexpansive mapping, that is, $\|S x-S y\| \leq\|x-y\|, \forall x, y \in \mathscr{H}$. Furthermore, $S$ is call a quasi-nonexpansive mapping if $\operatorname{Fix}(S) \neq \emptyset$ and $\|S x-y\| \leq\|x-y\|$, $\forall x \in \mathscr{H}, y \in \operatorname{Fix}(S)$. We remark here that the class of quasi-nonexpansive mappings is quite different with the class of nonexpansive mappings. Nonexpansive mappings are Lipschitz continuous, however, the class of quasi-nonexpansive mapping may be discontinuous. The theory of nonexpansive mappings finds a number of applications in convex optimization. Both the class of quasi-nonexpansive mappings and the class of nonexpansive mappings have been investigated extensively recently; see, e.g., $[1,2,3,4,5]$ and the references therein. Recall that $S$ is demiclosed at zero if for any sequence $\left\{x_{n}\right\}$ with $x_{n} \rightarrow x$, the implication holds: $S x_{k} \rightarrow 0 \Rightarrow S x=0$. Recall that a mapping $S$ is said to be monotone if $\langle S x-S y, x-y\rangle \geq 0$, $\forall x, y \in \mathscr{H} . S$ is said to be pseudomonotone if $\langle S x, y-x\rangle \geq 0 \Rightarrow\langle S y, y-x\rangle \geq 0, \forall x, y \in \mathscr{H} ; S$ is said to be strongly monotone if $\langle S x-S y, x-y\rangle \geq \beta\|x-y\|^{2}, \forall x, y \in \mathscr{H}$, where $\beta$ is some positive real number. It is also called a $\beta$-strongly monotone mapping.

E-mail address: zenglc@ @ shnu.edu.cn.

Received June 9, 2020; Accepted November 2, 2020.

(C)2021 Journal of Nonlinear and Variational Analysis 
Let $P_{C}$ be the metric projection from $\mathscr{H}$ onto $C$. It is known that $P_{C} x$ is nonexpansive and has the following basic properties:

(1) $\left\|P_{C} x-y\right\|^{2} \leq\|x-y\|^{2}-\left\|x-P_{C} x\right\|^{2}, \forall y \in C$;

(2) $\left\langle x-P_{C} x, y-P_{C} x\right\rangle \leq 0, \forall y \in C$;

(3) $\left\|P_{C} x-P_{C} y\right\|^{2} \leq\left\langle P_{C} x-P_{C} y, x-y\right\rangle, \forall y \in \mathscr{H}$.

Consider the classical variational inequality problem (VIP), which consists of finding $x^{*} \in C$ such that $\left\langle S x^{*}, x-x^{*}\right\rangle \geq 0, \forall x \in C$, where $S$ is a monotone mapping on $\mathscr{H}$. The solution set of the VIP is denoted by $\operatorname{Sol}(C, S)$. The VIP provide a unified framework for lots of problems arising in the real world; see, e.g., $[6,7,8,9,10]$ and the references therein. To the best of our knowledge, one of the most popular methods for solving the VIP is the extragradient method, first investigated by Korpelevich [11] for saddle point problems in 1976. This method is efficient for monotone VIPs with the aid of two projections. Recently, efforts and attention have been given to various extragradient-like methods; see, e.g, $[12,13,14,15,16]$ and the references therein.

To improve the efficiency of Korpelevich's extragradient method (due to the fact that two projection are involved), Censor, Gibali and Reich [17] first introduced the subgradient extragradient method, in which the second projection onto set $C$ is replaced by a projection onto a half-space, which is easy to implement

$$
\left\{\begin{array}{l}
x_{0} \in \mathscr{H} \\
y_{n}=P_{C}\left(x_{n}-\ell S x_{n}\right) \\
C_{n}=\left\{y \in \mathscr{H}:\left\langle x_{n}-\ell S x_{n}-y_{n}, y-y_{n}\right\rangle \leq 0\right\} \\
x_{n+1}=P_{C_{n}}\left(x_{n}-\ell S y_{n}\right), \quad \forall n \geq 0
\end{array}\right.
$$

where $S: \mathscr{H} \rightarrow \mathscr{H}$ is a monotone $\kappa$-Lipschitz mapping, and $\ell \in\left(0, \frac{1}{\kappa}\right)$. They proved that the sequence $\left\{x_{n}\right\}$ generated via the subgradient extragradient method converges weakly to a solution $x^{*} \in \operatorname{Sol}(C, S)$.

To accelerate the convergence rate of algorithms, much attention has been paid for developing various fast iterative algorithms from the viewpoint of numerical analysis recently; see, e.g., $[18,19,20,21,22]$ and the references therein. Among the recent acceleration methods, the inertial method, which is originated from physics, that is, a dynamical system that describes the behaviour of a rolling ball (heavy-ball dynamical system). The motion of the ball accelerates due to inertia. In 2018, Thong and Hieu [23] investigated an inertial subgradient extragradient algorithm and obtained the weak convergence of the algorithm. Recently, Thong and Hieu [24] suggested an inertial subgradient extragradient method with line-search process for solving the VIP with monotone and Lipschitz continuous mappings and the fixed point problem (FPP) of quasi-nonexpansive mappings.

On the other hand, let $\Theta: C \times C \rightarrow \mathbb{R}$ be a bifunction with $\Theta(x, x)=0, \forall x \in C$. Consider the equilibrium problem (EP) of finding $x^{*} \in C$ such that $\Theta\left(x^{*}, x\right) \geq 0, \forall x \in C$. We denote the solution set of the $\mathrm{EP}$ by $\operatorname{EP}(\Theta)$, i.e., $\operatorname{EP}(\Theta)=\left\{x^{*} \in C: \Theta\left(x^{*}, x\right) \geq 0, \forall x \in C\right\}$. It is worth mentioning that the EP is a unified model of several problems, namely, variational inequality problems, optimization problems, saddle point problems, complementarity problems, fixed point problems, Nash equilibrium problems and so forth. Many algorithms have been suggested and studied for solving the EP and its extensions; see, e.g., [25, 26, 27, 28, 29] and the therein references. Very recently, Dadashi, Iyiola and Shehu [26] suggested the following subgradient extragradient 
algorithm for solving the EP with a pseudomonotone, Lipschitz-type continuous bifunction. Under some mild conditions, they established the weak convergence of their algorithm in Hilbert spaces.

In this paper, we introduce modified inertial subgradient extragradient algorithms for solving the pseudomonotone EP with the constraint of finitely many nonexpansive mappings and one quasi-nonexpansive mapping in a real Hilbert space. In our algorithms, the subgradient projection onto some constructible half-space is used in place of the second minimization problem over a closed convex set, and a new approach for attaining the step-size is provided, that is, the step-sizes are not fixed but generated by our algorithms. Strong convergence theorems are proved under some suitable conditions.

\section{PRELIMINARIES}

Assume that $C$ is a nonempty closed convex subset of a real Hilbert space $\mathscr{H}$. The normal cone $N_{C}(u)$ of $C$ at $u \in C$ is defined by

$$
N_{C}(u)=\{w \in \mathscr{H}:\langle w, v-u\rangle \leq 0, \forall v \in C\} .
$$

The subdifferential of a convex function $g: C \rightarrow \mathbb{R}$ at $u \in C$ is defined by

$$
\partial g(u)=\{w \in \mathscr{H}: g(v)-g(u) \geq\langle w, v-u\rangle, \forall v \in C\} .
$$

Let $g: C \rightarrow \mathbb{R}$ be a convex subdifferentiable and lower semicontinuous function on $C$. Then, $x^{*} \in C$ is a solution to the convex minimization problem $\min \{g(x): x \in C\}$ if and only if $0 \in \partial g\left(x^{*}\right)+N_{C}\left(x^{*}\right)$.

Recall that a bifunction $\Theta: C \times C \rightarrow \mathbb{R}$ is said to be

(i) pseudomonotone on $C$ if $\Theta(x, y) \geq 0 \Rightarrow \Theta(y, x) \leq 0, \forall x, y \in C$;

(ii) Lipschitz-type continuous on $C$ if there exist two constants $c_{1}, c_{2}>0$ such that

$$
\Theta(x, y)+\Theta(y, z) \geq \Theta(x, z)-c_{1}\|x-y\|^{2}-c_{2}\|y-z\|^{2}, \quad \forall x, y, z \in C .
$$

We assume that the bifunction $\Theta: C \times C \rightarrow \mathbf{R}$ satisfies the following conditions:

(A1) $\Theta$ is pseudomonotone on $C$ and $\Theta(x, x)=0, \forall x \in C$;

(A2) $\Theta(x, \cdot)$ is convex and subdifferentiable on $H$ for every fixed $x \in H$;

(A3) $\Theta$ is jointly weakly continuous on $\Delta \times C$, where $\Delta$ is an open set containing $C$ in the sense that if $\left\{x_{k}\right\} \subset \Delta$ and $\left\{y_{k}\right\} \subset C$ are two sequences converging weakly to $x \in \Delta$ and $y \in C$, respectively, then $\Theta\left(x_{k}, y_{k}\right) \rightarrow \Theta(x, y)$ as $k \rightarrow \infty$;

(A4) $\Theta$ is Lipschitz-type continuous on $C$ with constants $c_{1}, c_{2}>0$.

It is easy to show that if the solution set $\operatorname{EP}(\Theta)$ is nonempty, then the solution set $\operatorname{EP}(\Theta)$ is closed and convex.

From the Lipschitz-type continuity of $\Theta$ on $C$ and [26, Lemma 2.5], we have the following result immediately.

Proposition 2.1. Let $C$ be a nonempty closed convex subset of a real Hilbert space $\mathscr{H}$. Let $\Theta$ be a Lipschitz-type continuous on $C$ with constants $c_{1}, c_{2}>0$. Suppose that $\rho \in(0,1), \delta<$ $\min \left\{1, \frac{1}{2 c_{1}}, \frac{1}{2 C_{2}}\right\}$ and $0<\mu<\delta$. Then, there exists a real number $\lambda$ such that $\mu \Theta(y, z) \geq$ $\lambda\left(\Theta(x, z)-\Theta(x, y)-c_{1}\|x-y\|^{2}-c_{2}\|y-z\|^{2}\right)$ and $\rho \mu<\lambda<\delta$, where $x, y, z \in C$.

In addition, we also need the following lemmas for our main result. 
Lemma 2.1. [30] Let $C$ be a nonempty closed convex subset of a real Hilbert space $\mathscr{H}$. Let $A: C \rightarrow \mathscr{H}$ be a pseudomonotone and continuous mapping and let $x \in C$. Then $\langle A x, y-x\rangle \geq 0$, $\forall y \in C \Leftrightarrow\langle A y, y-x\rangle \geq 0, \forall y \in C$.

Lemma 2.2. [31] Let $C$ be a nonempty closed convex subset of a real Hilbert space $\mathscr{H}$. Let $\lambda$ be a real number in $(0,1]$, and let $T: C \rightarrow \mathscr{H}$ be a nonexpansive mapping. Let $T^{\lambda}: C \rightarrow H$ be a mapping defined by $T^{\lambda} x:=T x-\lambda v F(T x), \forall x \in C$, where $F: H \rightarrow H$ is $\kappa$-Lipschitzian and $\eta$-strongly monotone. Then $T^{\lambda}$ is a contraction provided $0<v<\frac{2 \eta}{\kappa^{2}}$, i.e., $\left\|T^{\lambda} x-T^{\lambda} y\right\| \leq$ $(1-\lambda \tau)\|x-y\|, \forall x, y \in C$, where $\tau=1-\sqrt{1-v\left(2 \eta-v \kappa^{2}\right)} \in(0,1]$.

Lemma 2.3. [32] Let $C$ be a nonempty closed convex subset of a real Hilbert space $\mathscr{H}$. Let $\Gamma$ be a nonexpansive self-mapping on $C$ with $\operatorname{Fix}(\Gamma) \neq \emptyset$. Then $I-\Gamma$ is demiclosed at zero, that is, if $\left\{x_{k}\right\}$ is a sequence in $C$ such that $x_{k} \rightarrow x \in C$ and $(I-\Gamma) x_{k} \rightarrow 0$, then $(I-\Gamma) x=0$, where $I$ is the identity mapping of $\mathscr{H}$.

Lemma 2.4. [31] Let $\left\{a_{k}\right\}$ be a real sequence in $[0,+\infty)$ satisfying the condition: $a_{k+1} \leq$ $\left(1-s_{k}\right) a_{k}+s_{k} b_{k}, \forall k \geq 0$, where $\left\{s_{k}\right\}$ and $\left\{b_{k}\right\}$ lie in $\mathbf{R}:=(-\infty, \infty)$ such that $(a)\left\{s_{k}\right\} \subset[0,1]$ and $\sum_{k=0}^{\infty} s_{k}=\infty$, and (b) $\lim \sup _{k \rightarrow \infty} b_{k} \leq 0$ or $\sum_{k=0}^{\infty}\left|s_{k} b_{k}\right|<\infty$. Then $\lim _{k \rightarrow \infty} a_{k}=0$.

Lemma 2.5. [33] Let $\left\{\Gamma_{n}\right\}$ be a sequence of real numbers that does not decrease at infinity in the sense that there exists a subsequence $\left\{\Gamma_{n_{k}}\right\}$ of $\left\{\Gamma_{n}\right\}$ which satisfies $\Gamma_{n_{k}}<\Gamma_{n_{k}+1}$ for each integer $k \geq 1$. Define the sequence $\{\tau(n)\}_{n \geq n_{0}}$ of integers as follows:

$$
\tau(n)=\max \left\{k \leq n: \Gamma_{k}<\Gamma_{k+1}\right\},
$$

where integer $n_{0} \geq 1$ such that $\left\{k \leq n_{0}: \Gamma_{k}<\Gamma_{k+1}\right\} \neq \emptyset$. Then, the following hold:

(i) $\tau\left(n_{0}\right) \leq \tau\left(n_{0}+1\right) \leq \cdots$ and $\tau(n) \rightarrow \infty$;

(ii) $\Gamma_{\tau(n)} \leq \Gamma_{\tau(n)+1}$ and $\Gamma_{n} \leq \Gamma_{\tau(n)+1}, \forall n \geq n_{0}$.

\section{MAin Results}

In this section, we present our main algorithms and analyze their convergence. Throughout this section, we always assume that the following conditions hold:

$T_{i}$ is a nonexpansive mapping on $\mathscr{H}$ for $i=1, \ldots, N, T$ is a quasi-nonexpansive mapping on $\mathscr{H}$ with the fact that $I-T$ is demiclosed at zero;

$\Theta$ satisfies conditions (A1)-(A4) such that $\Omega=\bigcap_{i=0}^{N} \operatorname{Fix}\left(T_{i}\right) \cap \operatorname{EP}(\Theta) \neq \emptyset$, where $T_{0}:=T$;

$f: H \rightarrow H$ is a contraction with constant $\zeta \in[0,1)$, and $F: \mathscr{H} \rightarrow \mathscr{H}$ is $\eta$-strongly monotone and $\kappa$-Lipschitzian such that $\zeta<\tau:=1-\sqrt{1-v\left(2 \eta-v \kappa^{2}\right)}$ for $v \in\left(0, \frac{2 \eta}{\kappa^{2}}\right)$;

$\left\{\varepsilon_{n}\right\} \subset(0, \infty)$, and $\left\{\beta_{n}\right\},\left\{\gamma_{n}\right\},\left\{\sigma_{n}\right\} \subset(0,1)$ are sequences such that

(i) $\beta_{n}+\gamma_{n} \leq 1, \sum_{n=1}^{\infty} \beta_{n}=\infty$ and $\lim _{n \rightarrow \infty} \beta_{n}=0$;

(ii) $0<\liminf _{n \rightarrow \infty} \gamma_{n} \leq \limsup _{n \rightarrow \infty} \gamma_{n}<1$ and $0<\liminf _{n \rightarrow \infty} \sigma_{n} \leq \limsup _{n \rightarrow \infty} \sigma_{n}<1$;

(iii) $\varepsilon_{n}=o\left(\beta_{n}\right)$, i.e., $\lim _{n \rightarrow \infty} \frac{\varepsilon_{n}}{\beta_{n}}=0$.

In addition, we write $T_{n}:=T_{n \bmod N}$ for integer $n \geq 1$ with the mod function taking values in the set $\{1,2, \ldots, N\}$, that is, if $n=j N+q$ for some integers $j \geq 0$ and $0 \leq q<N$, then $T_{n}=T_{N}$, if $q=0$, and $T_{n}=T_{q}$ if $0<q<N$.

Algorithm 3.1. Initial Step: Let $\lambda_{1}>0, \alpha>0, \rho \in(0,1), \delta<\min \left\{1, \frac{1}{2 c_{1}}, \frac{1}{2 c_{2}}\right\}, 0<\mu<\delta$ and $x_{0}, x_{1} \in H$ be arbitrary.

Iterative Steps: Calculate $x_{n+1}$ as follows: 
Step 1. Given the iterates $x_{n-1}$ and $x_{n}(n \geq 1)$, choose $\alpha_{n}$ such that $0 \leq \alpha_{n} \leq \bar{\alpha}_{n}$, where

$$
\bar{\alpha}_{n}=\left\{\begin{array}{lc}
\min \left\{\alpha, \frac{\varepsilon_{n}}{\left\|x_{n}-x_{n-1}\right\|}\right\}, & \text { if } x_{n} \neq x_{n-1}, \\
\alpha, & \text { otherwise. }
\end{array}\right.
$$

Step 2. Compute $p_{n}=x_{n}+\alpha_{n}\left(x_{n}-x_{n-1}\right), v_{n}=\sigma_{n} p_{n}+\left(1-\sigma_{n}\right) T_{n} p_{n}$ and

$$
y_{n}=\operatorname{argmin}\left\{\lambda_{n} \Theta\left(p_{n}, y\right)+\frac{1}{2}\left\|p_{n}-y\right\|^{2}: y \in C\right\} .
$$

Step 3. Pick $w_{n} \in \partial_{2} \Theta\left(p_{n}, \cdot\right) y_{n}$, construct $C_{n}=\left\{z \in H:\left\langle\left(p_{n}-\lambda_{n} w_{n}\right)-y_{n}, z-y_{n}\right\rangle \leq 0\right\}$ (i.e., a half-space whose bounding hyperplane supports $C$ at $\left.y_{n}\right)$, and compute $z_{n}=\operatorname{argmin}\left\{\mu \lambda_{n} \Theta\left(y_{n}, y\right)+\right.$ $\left.\frac{1}{2}\left\|p_{n}-y\right\|^{2}: y \in C_{n}\right\}$.

Step 4. Calculate $x_{n+1}=\beta_{n} f\left(z_{n}\right)+\gamma_{n} v_{n}+\left(\left(1-\gamma_{n}\right) I-\beta_{n} v F\right) T z_{n}$, and update

$$
\lambda_{n+1}=\min \left\{\delta, \frac{\mu \Theta\left(y_{n}, z_{n}\right)}{\Theta\left(p_{n}, z_{n}\right)-\Theta\left(p_{n}, y_{n}\right)-c_{1}\left\|p_{n}-y_{n}\right\|^{2}-c_{2}\left\|y_{n}-z_{n}\right\|^{2}+1}\right\} .
$$

Set $n:=n+1$ and return to Step 1 .

Remark 3.1. (i) From (3.1), it follows that $\lim _{n \rightarrow \infty} \frac{\alpha_{n}}{\beta_{n}}\left\|x_{n}-x_{n-1}\right\|=0$. Indeed, we have $\alpha_{n} \| x_{n}-$ $x_{n-1} \| \leq \varepsilon_{n}, \forall n \geq 1$, which together with $\lim _{n \rightarrow \infty} \frac{\varepsilon_{n}}{\beta_{n}}=0$ implies that

$$
\lim _{n \rightarrow \infty} \frac{\alpha_{n}}{\beta_{n}}\left\|x_{n}-x_{n-1}\right\| \leq \lim _{n \rightarrow \infty} \frac{\varepsilon_{n}}{\beta_{n}}=0 .
$$

(ii) It is easy to see that $p_{n} \in \operatorname{EP}(\Theta) \Leftrightarrow p_{n}=y_{n}$. In our convergence analysis, we assume that $p_{n} \neq y_{n}$. According to Proposition 2.1, we have that $\lambda_{n+1}$ in (3.2) is well-defined and

$$
\mu \Theta\left(y_{n}, z_{n}\right) \geq \lambda_{n+1}\left(\Theta\left(p_{n}, z_{n}\right)-\Theta\left(p_{n}, y_{n}\right)-c_{1}\left\|p_{n}-y_{n}\right\|^{2}-c_{2}\left\|y_{n}-z_{n}\right\|^{2}\right) .
$$

We are now in a position to state and prove the first main result in this paper.

Theorem 3.1. Let $C$ be a nonempty closed convex subset of a real Hilbert space $\mathscr{H}$. Let $\left\{x_{n}\right\}$ be the sequence constructed by Algorithm 3.1. Then $x_{n} \rightarrow x^{*} \in \Omega$, which is a unique solution to the VIP: $\left\langle(v F-f) x^{*}, p-x^{*}\right\rangle \geq 0, \forall p \in \Omega$.

Proof. From Lemma 2.2, we have

$$
\begin{aligned}
& \left\|P_{\Omega}(f+I-v F) x-P_{\Omega}(f+I-v F) y\right\| \\
& \leq\|f(x)-f(y)\|+\|(I-v F) x-(I-v F) y\| \\
& \leq[1-(\tau-\zeta)]\|x-y\| \quad \forall x, y \in \mathscr{H}
\end{aligned}
$$

which implies that $P_{\Omega}(f+I-v F)$ is a contraction. Banach contraction principle guarantees that $P_{\Omega}(f+I-v F)$ has a unique fixed point, say $x^{*} \in H$, that is, $x^{*}=P_{\Omega}(f+I-v F) x^{*}$. Thus, there exists a unique solution $x^{*} \in \Omega$ to the $\operatorname{VIP}\left\langle(v F-f) x^{*}, p-x^{*}\right\rangle \geq 0, \forall p \in \Omega$.

Next, we divide the rest of the proof into several steps.

Step 1. Show

$$
\begin{aligned}
\left\|z_{n}-x^{*}\right\|^{2} \leq & \left\|p_{n}-x^{*}\right\|^{2}-\left(1-\lambda_{n+1}\right)\left\|p_{n}-z_{n}\right\|^{2}-\lambda_{n+1}\left(1-2 \lambda_{n} c_{1}\right)\left\|p_{n}-y_{n}\right\|^{2} \\
& -\lambda_{n+1}\left(1-2 \lambda_{n} c_{2}\right)\left\|z_{n}-y_{n}\right\|^{2}
\end{aligned}
$$

From the definition of $z_{n}$ and Proposition 2.1, we get

$$
0 \in \partial_{2}\left(\mu \lambda_{n} \Theta\left(y_{n}, y\right)+\frac{1}{2}\left\|p_{n}-y\right\|^{2}\right)\left(z_{n}\right)+N_{C_{n}}\left(z_{n}\right) .
$$


Hence, there exist $w \in \partial_{2} \Theta\left(y_{n}, \cdot\right)\left(z_{n}\right)$ and $\bar{w} \in N_{C_{n}}\left(z_{n}\right)$ such that $\mu \lambda_{n} w+z_{n}-p_{n}+\bar{w}=0$. Thus,

$$
\left\langle z_{n}-p_{n}, y-z_{n}\right\rangle=\mu \lambda_{n}\left\langle w, z_{n}-y\right\rangle+\left\langle\bar{w}, z_{n}-y\right\rangle .
$$

Since $\bar{w} \in N_{C_{n}}\left(z_{n}\right)$, we obtain that $\left\langle\bar{w}, y-z_{n}\right\rangle \leq 0, \forall y \in C_{n}$. This together with (3.5) implies that $\left\langle z_{n}-p_{n}, y-z_{n}\right\rangle \geq \mu \lambda_{n}\left\langle w, z_{n}-y\right\rangle, \forall y \in C_{n}$. From $w \in \partial_{2} \Theta\left(y_{n}, \cdot\right)\left(z_{n}\right)$, we have $\left\langle w, z_{n}-y\right\rangle \geq$ $\Theta\left(y_{n}, z_{n}\right)-\Theta\left(y_{n}, y\right), \forall y \in \mathscr{H}$. By the last two inequalities, we have

$$
\left\langle z_{n}-p_{n}, y-z_{n}\right\rangle \geq \mu \lambda_{n}\left(\Theta\left(y_{n}, z_{n}\right)-\Theta\left(y_{n}, y\right)\right), \quad \forall y \in C_{n}
$$

In view of $x^{*} \in \operatorname{EP}(\Omega)$ and $y_{n} \in C$, we have $\Theta\left(x^{*}, y_{n}\right) \geq 0$. Hence, it follows from the pseudomonotonicity of $\Theta$ that $\Theta\left(y_{n}, x^{*}\right) \leq 0$. This together with (3.6) ensures that

$$
\left\langle z_{n}-p_{n}, x^{*}-z_{n}\right\rangle \geq \mu \lambda_{n} \Theta\left(y_{n}, z_{n}\right) .
$$

From $w_{n} \in \partial_{2} \Theta\left(p_{n}, \cdot\right) y_{n}$, we get $\left\langle w_{n}, y-y_{n}\right\rangle \leq \Theta\left(p_{n}, y\right)-\Theta\left(p_{n}, y_{n}\right), \forall y \in \mathscr{H}$. Setting $y=z_{n}$ in the inequality above, we arrive at

$$
\left\langle w_{n}, z_{n}-y_{n}\right\rangle \leq \Theta\left(p_{n}, z_{n}\right)-\Theta\left(p_{n}, y_{n}\right), \quad \forall y \in \mathscr{H} .
$$

From $z_{n} \in C_{n}$, it follows that

$$
\left\langle p_{n}-y_{n}, z_{n}-y_{n}\right\rangle \leq \lambda_{n}\left\langle w_{n}, z_{n}-y_{n}\right\rangle
$$

which together with (3.8) implies

$$
\left\langle p_{n}-y_{n}, z_{n}-y_{n}\right\rangle \leq \lambda_{n}\left(\Theta\left(p_{n}, z_{n}\right)-\Theta\left(p_{n}, y_{n}\right)\right) .
$$

Consequently, combining (3.7) and (3.9) yields that

$$
\begin{aligned}
& 2 \lambda_{n+1}\left[\left\langle p_{n}-y_{n}, z_{n}-y_{n}\right\rangle-\lambda_{n}\left(c_{1}\left\|p_{n}-y_{n}\right\|^{2}+c_{2}\left\|y_{n}-z_{n}\right\|^{2}\right)\right] \\
& \leq 2 \lambda_{n} \lambda_{n+1}\left[\Theta\left(p_{n}, z_{n}\right)-\Theta\left(p_{n}, y_{n}\right)-c_{1}\left\|p_{n}-y_{n}\right\|^{2}-c_{2}\left\|y_{n}-z_{n}\right\|^{2}\right] \\
& \leq 2 \mu \lambda_{n} \Theta\left(y_{n}, z_{n}\right) \\
& \leq 2\left\langle z_{n}-p_{n}, x^{*}-z_{n}\right\rangle .
\end{aligned}
$$

It follows that

$$
\begin{aligned}
& \lambda_{n+1}\left(\left\|p_{n}-y_{n}\right\|^{2}+\left\|z_{n}-y_{n}\right\|^{2}-\left\|p_{n}-z_{n}\right\|^{2}\right) \\
& \leq\left\|p_{n}-x^{*}\right\|^{2}-\left\|z_{n}-p_{n}\right\|^{2}-\left\|z_{n}-x^{*}\right\|^{2}+2 \lambda_{n} \lambda_{n+1} c_{1}\left\|p_{n}-y_{n}\right\|^{2}+2 \lambda_{n} \lambda_{n+1} c_{2}\left\|y_{n}-z_{n}\right\|^{2} .
\end{aligned}
$$

This leads to the desired conclusion.

Step 2. Show that $\left\{x_{n}\right\}$ is bounded.

Indeed, since $1>\limsup _{n \rightarrow \infty} \gamma_{n} \geq \liminf _{n \rightarrow \infty} \gamma_{n}>0$ and $1>\limsup _{n \rightarrow \infty} \sigma_{n} \geq \liminf _{n \rightarrow \infty} \sigma_{n}>$ 0 , we may assume, without loss of generality, that $\left\{\gamma_{n}\right\} \subset[a, b] \subset(0,1)$ and $\left\{\sigma_{n}\right\} \subset[c, d] \subset(0,1)$. From $x^{*} \in \Omega=\bigcap_{i=0}^{N} \operatorname{Fix}\left(T_{i}\right) \cap \operatorname{EP}(\Theta)$, we get $T x^{*}=x^{*}, T_{n} x^{*}=x^{*}$ and $x^{*} \in \operatorname{EP}(\Theta)$. From Proposition 2.1, we get $\rho \mu<\lambda_{n}<\delta$. Then, it follows from (3.4) that

$$
\left\|z_{n}-x^{*}\right\| \leq\left\|p_{n}-x^{*}\right\| \text {. }
$$

It follows from the definition of $p_{n}$ that

$$
\begin{aligned}
\left\|p_{n}-x^{*}\right\| & =\left\|x_{n}+\alpha_{n}\left(x_{n}-x_{n-1}\right)-x^{*}\right\| \\
& \leq\left\|x_{n}-x^{*}\right\|+\beta_{n} \cdot \frac{\alpha_{n}}{\beta_{n}}\left\|x_{n}-x_{n-1}\right\| .
\end{aligned}
$$


According to (3.3), we have $\frac{\alpha_{n}}{\beta_{n}}\left\|x_{n}-x_{n-1}\right\| \rightarrow 0(n \rightarrow \infty)$. So, there exists a constant $M_{0}>0$ such that

$$
\frac{\alpha_{n}}{\beta_{n}}\left\|x_{n}-x_{n-1}\right\| \leq M_{0}
$$

Combining (3.10), (3.11) and (3.12), we arrive at

$$
\left\|z_{n}-x^{*}\right\| \leq\left\|p_{n}-x^{*}\right\| \leq\left\|x_{n}-x^{*}\right\|+\beta_{n} M_{0}
$$

In view of $v_{n}=\sigma_{n} p_{n}+\left(1-\sigma_{n}\right) T_{n} p_{n}$, we get

$$
\left\|v_{n}-x^{*}\right\| \leq \sigma_{n}\left\|p_{n}-x^{*}\right\|+\left(1-\sigma_{n}\right)\left\|T_{n} p_{n}-x^{*}\right\| \leq\left\|p_{n}-x^{*}\right\| .
$$

Therefore, from $\beta_{n}+\gamma_{n} \leq 1$, Lemma 2.2 and (3.13), we conclude that

$$
\begin{aligned}
\left\|x_{n+1}-x^{*}\right\| \leq & \beta_{n}\left(\left\|f\left(z_{n}\right)-f\left(x^{*}\right)\right\|+\left\|f\left(x^{*}\right)-x^{*}\right\|\right)+\gamma_{n}\left\|v_{n}-x^{*}\right\| \\
& +\left(1-\beta_{n}-\gamma_{n}\right)\left\|\left(\frac{1-\gamma_{n}}{1-\beta_{n}-\gamma_{n}} I-\frac{\beta_{n}}{1-\beta_{n}-\gamma_{n}} v F\right) T z_{n}-x^{*}\right\| \\
\leq & \beta_{n}\left(\zeta\left\|z_{n}-x^{*}\right\|+\left\|f\left(x^{*}\right)-x^{*}\right\|\right)+\gamma_{n}\left\|p_{n}-x^{*}\right\| \\
& +\left(1-\gamma_{n}\right)\left\|\left(I-\frac{\beta_{n}}{1-\gamma_{n}} v F\right) T z_{n}-\left(I-\frac{\beta_{n}}{1-\gamma_{n}} v F\right) x^{*}+\frac{\beta_{n}}{1-\gamma_{n}}(I-v F) x^{*}\right\| \\
\leq & \beta_{n}\left(\zeta\left\|z_{n}-x^{*}\right\|+\left\|f\left(x^{*}\right)-x^{*}\right\|\right)+\gamma_{n}\left\|p_{n}-x^{*}\right\| \\
& +\left(1-\gamma_{n}\right)\left[\left(1-\frac{\beta_{n}}{1-\gamma_{n}} \tau\right)\left\|z_{n}-x^{*}\right\|+\frac{\beta_{n}}{1-\gamma_{n}}\left\|(I-v F) x^{*}\right\|\right] \\
\leq & {\left[1-\beta_{n}(\tau-\zeta)\right]\left(\left\|x_{n}-x^{*}\right\|+\beta_{n} M_{0}\right)+\beta_{n}\left(\left\|f\left(x^{*}\right)-x^{*}\right\|+\left\|(I-v F) x^{*}\right\|\right) } \\
\leq & {\left[1-\beta_{n}(\tau-\zeta)\right]\left\|x_{n}-x^{*}\right\|+\beta_{n}\left(M_{0}+\left\|f\left(x^{*}\right)-x^{*}\right\|+\left\|(I-v F) x^{*}\right\|\right) } \\
= & {\left[1-\beta_{n}(\tau-\zeta)\right]\left\|x_{n}-x^{*}\right\|+\beta_{n}(\tau-\zeta) \cdot \frac{M_{0}+\left\|f\left(x^{*}\right)-x^{*}\right\|+\left\|(I-v F) x^{*}\right\|}{\tau-\zeta} } \\
\leq & \max \left\{\left\|x_{n}-x^{*}\right\|, \frac{M_{0}+\left\|f\left(x^{*}\right)-x^{*}\right\|+\left\|(I-v F) x^{*}\right\|}{\tau-\zeta}\right\} .
\end{aligned}
$$

By induction, we obtain

$$
\left\|x_{n}-x^{*}\right\| \leq \max \left\{\left\|x_{1}-x^{*}\right\|, \frac{M_{0}+\left\|f\left(x^{*}\right)-x^{*}\right\|+\left\|(I-v F) x^{*}\right\|}{\tau-\zeta}\right\}, \quad \forall n \geq 1
$$

Thus, $\left\{x_{n}\right\}$ is bounded, so are $\left\{p_{n}\right\},\left\{z_{n}\right\},\left\{f\left(z_{n}\right)\right\},\left\{T_{n} p_{n}\right\}$, and $\left\{T z_{n}\right\}$.

Step 3. Show that $\left\{x_{n}\right\}$ converges strongly to $x^{*}$. 
Indeed, utilizing Lemma 2.2, (3.10), the convexity of the function $h(s)=s^{2}, \forall s \in \mathbb{R}$, we obtain that

$$
\begin{aligned}
\left\|x_{n+1}-x^{*}\right\|^{2}= & \| \beta_{n}\left(f\left(z_{n}\right)-f\left(x^{*}\right)\right)+\gamma_{n}\left(v_{n}-x^{*}\right)+\left(1-\gamma_{n}\right)\left[\left(I-\frac{\beta_{n}}{1-\gamma_{n}} v F\right) T z_{n}\right. \\
& \left.-\left(I-\frac{\beta_{n}}{1-\gamma_{n}} v F\right) x^{*}\right]+\beta_{n}(f-v F) x^{*} \|^{2} \\
\leq & \| \beta_{n}\left(f\left(z_{n}\right)-f\left(x^{*}\right)\right)+\gamma_{n}\left(v_{n}-x^{*}\right)+\left(1-\gamma_{n}\right)\left[\left(I-\frac{\beta_{n}}{1-\gamma_{n}} v F\right) T z_{n}\right. \\
& \left.-\left(I-\frac{\beta_{n}}{1-\gamma_{n}} v F\right) x^{*}\right] \|^{2}+2 \beta_{n}\left\langle(f-v F) x^{*}, x_{n+1}-x^{*}\right\rangle \\
\leq & {\left[\beta_{n} \zeta\left\|z_{n}-x^{*}\right\|+\gamma_{n}\left\|p_{n}-x^{*}\right\|+\left(1-\beta_{n} \tau-\gamma_{n}\right)\left\|z_{n}-x^{*}\right\|\right]^{2} } \\
& +2 \beta_{n}\left\langle(f-v F) x^{*}, x_{n+1}-x^{*}\right\rangle \\
\leq & \beta_{n} \zeta\left\|z_{n}-x^{*}\right\|^{2}+\gamma_{n}\left\|p_{n}-x^{*}\right\|^{2}+\left(1-\beta_{n} \tau-\gamma_{n}\right)\left\|z_{n}-x^{*}\right\|^{2} \\
& +2 \beta_{n}\left\langle(f-v F) x^{*}, x_{n+1}-x^{*}\right\rangle \\
\leq & {\left[1-\beta_{n}(\tau-\zeta)\right]\left\|p_{n}-x^{*}\right\|^{2}+2 \beta_{n}\left\langle(f-v F) x^{*}, x_{n+1}-x^{*}\right\rangle . }
\end{aligned}
$$

due to $\beta_{n} \zeta+\gamma_{n}+\left(1-\beta_{n} \tau-\gamma_{n}\right)=1-\beta_{n}(\tau-\zeta) \leq 1$. We claim that

$$
\begin{aligned}
\left\|x_{n+1}-x^{*}\right\|^{2} \leq & {\left[1-\beta_{n}(\tau-\zeta)\right]\left\|x_{n}-x^{*}\right\|^{2} } \\
& +\beta_{n}(\tau-\zeta)\left[\frac{2}{\tau-\zeta}\left\langle(f-v F) x^{*}, x_{n+1}-x^{*}\right\rangle+\frac{3 M}{\tau-\zeta} \cdot \frac{\alpha_{n}}{\beta_{n}}\left\|x_{n}-x_{n-1}\right\|\right],
\end{aligned}
$$

for some $M>0$. In fact, we get

$$
\left\|p_{n}-x^{*}\right\|^{2} \leq\left\|x_{n}-x^{*}\right\|^{2}+\alpha_{n}\left\|x_{n}-x_{n-1}\right\|\left[2\left\|x_{n}-x^{*}\right\|+\alpha_{n}\left\|x_{n}-x_{n-1}\right\|\right] .
$$

Combining (3.14) and (3.15), we deduce that

$$
\begin{aligned}
\left\|x_{n+1}-x^{*}\right\|^{2} \leq & {\left[1-\beta_{n}(\tau-\zeta)\right]\left\|p_{n}-x^{*}\right\|^{2}+2 \beta_{n}\left\langle(f-v F) x^{*}, x_{n+1}-x^{*}\right\rangle } \\
\leq & {\left[1-\beta_{n}(\tau-\zeta)\right]\left\{\left\|x_{n}-x^{*}\right\|^{2}+\alpha_{n}\left\|x_{n}-x_{n-1}\right\|\left[2\left\|x_{n}-x^{*}\right\|+\alpha_{n}\left\|x_{n}-x_{n-1}\right\|\right]\right\} } \\
& +2 \beta_{n}\left\langle(f-v F) x^{*}, x_{n+1}-x^{*}\right\rangle \\
\leq & {\left[1-\beta_{n}(\tau-\zeta)\right]\left\|x_{n}-x^{*}\right\|^{2}+\alpha_{n}\left\|x_{n}-x_{n-1}\right\|\left[2\left\|x_{n}-x^{*}\right\|+\alpha_{n}\left\|x_{n}-x_{n-1}\right\|\right] } \\
& +2 \beta_{n}\left\langle(f-v F) x^{*}, x_{n+1}-x^{*}\right\rangle \\
\leq & {\left[1-\beta_{n}(\tau-\zeta)\right]\left\|x_{n}-x^{*}\right\|^{2}+\alpha_{n}\left\|x_{n}-x_{n-1}\right\| 3 M+2 \beta_{n}\left\langle(f-v F) x^{*}, x_{n+1}-x^{*}\right\rangle } \\
= & {\left[1-\beta_{n}(\tau-\zeta)\right]\left\|x_{n}-x^{*}\right\|^{2} } \\
& +\beta_{n}(\tau-\zeta)\left[\frac{2\left\langle(f-v F) x^{*}, x_{n+1}-x^{*}\right\rangle}{\tau-\zeta}+\frac{3 M}{\tau-\zeta} \frac{\alpha_{n}}{\beta_{n}}\left\|x_{n}-x_{n-1}\right\|\right],
\end{aligned}
$$

where $\sup _{n \geq 1}\left\{\left\|x_{n}-x^{*}\right\|, \alpha_{n}\left\|x_{n}-x_{n-1}\right\|\right\} \leq M$ for some $M>0$. For each $n \geq 1$, we set $\Gamma_{n}=$ $\left\|x_{n}-x^{*}\right\|^{2}, \varepsilon_{n}=\beta_{n}(\tau-\zeta)$ and $\vartheta_{n}=\alpha_{n}\left\|x_{n}-x_{n-1}\right\| 3 M+2 \beta_{n}\left\langle(f-v F) x^{*}, x_{n+1}-x^{*}\right\rangle$. Then (3.16) can be rewritten as the following formula $\Gamma_{n+1} \leq\left(1-\varepsilon_{n}\right) \Gamma_{n}+\vartheta_{n}$.

Finally, we show the convergence of $\left\{\Gamma_{n}\right\}$ to zero by the following two cases:

Case 1. Suppose that there exists a positive integer $n_{0}$ such that $\left\{\Gamma_{n}\right\}$ is non-increasing for $n \geq n_{0}$. Then we obtain that the $\operatorname{limit}_{n \rightarrow \infty}\left\|\lim _{n}-x^{*}\right\|$ exists and $\Gamma_{n}-\Gamma_{n+1} \rightarrow 0$ as $n \rightarrow \infty$. So, 
it follows from (3.4), (3.14) and (3.15) that

$$
\begin{aligned}
& \left\|x_{n+1}-x^{*}\right\|^{2} \\
\leq & {\left[1-\beta_{n}(\tau-\zeta)-\gamma_{n}\right]\left\|z_{n}-x^{*}\right\|^{2}+\gamma_{n}\left\|p_{n}-x^{*}\right\|^{2}+2 \beta_{n}\left\langle(f-v F) x^{*}, x_{n+1}-x^{*}\right\rangle } \\
\leq & {\left[1-\beta_{n}(\tau-\zeta)-\gamma_{n}\right]\left[\left\|p_{n}-x^{*}\right\|^{2}-\left(1-\lambda_{n+1}\right)\left\|p_{n}-z_{n}\right\|^{2}-\lambda_{n+1}\left(1-2 \lambda_{n} c_{1}\right)\left\|p_{n}-y_{n}\right\|^{2}\right.} \\
& \left.-\lambda_{n+1}\left(1-2 \lambda_{n} c_{2}\right)\left\|z_{n}-y_{n}\right\|^{2}\right]+\gamma_{n}\left\|p_{n}-x^{*}\right\|^{2}+2 \beta_{n}\left\|(f-v F) x^{*}\right\|\left\|x_{n+1}-x^{*}\right\| \\
\leq & {\left[1-\beta_{n}(\tau-\zeta)\right]\left\|p_{n}-x^{*}\right\|^{2}-\left[1-\beta_{n}(\tau-\zeta)-\gamma_{n}\right]\left[\left(1-\lambda_{n+1}\right)\left\|p_{n}-z_{n}\right\|^{2}\right.} \\
& \left.+\lambda_{n+1}\left(1-2 \lambda_{n} c_{1}\right)\left\|p_{n}-y_{n}\right\|^{2}+\lambda_{n+1}\left(1-2 \lambda_{n} c_{2}\right)\left\|z_{n}-y_{n}\right\|^{2}\right]+\beta_{n} M_{1} \\
\leq & \left\|x_{n}-x^{*}\right\|^{2}+\alpha_{n}\left\|x_{n}-x_{n-1}\right\| 3 M-\left[1-\beta_{n}(\tau-\zeta)-\gamma_{n}\right]\left[\left(1-\lambda_{n+1}\right)\left\|p_{n}-z_{n}\right\|^{2}\right. \\
& \left.+\lambda_{n+1}\left(1-2 \lambda_{n} c_{1}\right)\left\|p_{n}-y_{n}\right\|^{2}+\lambda_{n+1}\left(1-2 \lambda_{n} c_{2}\right)\left\|z_{n}-y_{n}\right\|^{2}\right]+\beta_{n} M_{1},
\end{aligned}
$$

where $\sup _{n \geq 1} 2\left\|(f-v F) x^{*}\right\|\left\|x_{n}-x^{*}\right\| \leq M_{1}$ for some $M_{1}>0$. This immediately yields

$$
\begin{aligned}
& {\left[1-\beta_{n}(\tau-\zeta)-\gamma_{n}\right]\left[\left(1-\lambda_{n+1}\right)\left\|p_{n}-z_{n}\right\|^{2}+\lambda_{n+1}\left(1-2 \lambda_{n} c_{1}\right)\left\|p_{n}-y_{n}\right\|^{2}\right.} \\
& \left.+\lambda_{n+1}\left(1-2 \lambda_{n} c_{2}\right)\left\|z_{n}-y_{n}\right\|^{2}\right] \leq \Gamma_{n}-\Gamma_{n+1}+\alpha_{n}\left\|x_{n}-x_{n-1}\right\| 3 M+\beta_{n} M_{1} .
\end{aligned}
$$

Since $\Gamma_{n}-\Gamma_{n+1} \rightarrow 0, \beta_{n} \rightarrow 0, \alpha_{n}\left\|x_{n}-x_{n-1}\right\| \rightarrow 0$ and $\liminf _{n \rightarrow \infty}\left(1-\gamma_{n}\right)>0$, we obtain from $\rho \mu<\lambda_{n}<\delta(\forall n>1)$ that

$$
\lim _{n \rightarrow \infty}\left\|p_{n}-z_{n}\right\|=\lim _{n \rightarrow \infty}\left\|p_{n}-y_{n}\right\|=\lim _{n \rightarrow \infty}\left\|z_{n}-y_{n}\right\|=0
$$

Since $p_{n}=x_{n}+\alpha_{n}\left(x_{n}-x_{n-1}\right)$, we get

$$
\lim _{n \rightarrow \infty}\left\|p_{n}-x_{n}\right\|=\lim _{n \rightarrow \infty} \alpha_{n}\left\|x_{n}-x_{n-1}\right\|=0 .
$$

It follows from (3.13) that

$$
\begin{aligned}
\left\|x_{n+1}-x^{*}\right\|^{2}= & \left\|\beta_{n}\left(f\left(z_{n}\right)-v F T z_{n}\right)+\gamma_{n}\left(v_{n}-x^{*}\right)+\left(1-\gamma_{n}\right)\left(T z_{n}-x^{*}\right)\right\|^{2} \\
\leq & \left\|\gamma_{n}\left(v_{n}-x^{*}\right)+\left(1-\gamma_{n}\right)\left(T z_{n}-x^{*}\right)\right\|^{2}+2 \beta_{n}\left\langle f\left(z_{n}\right)-v F T z_{n}, x_{n+1}-x^{*}\right\rangle \\
= & \gamma_{n}\left\|v_{n}-x^{*}\right\|^{2}+\left(1-\gamma_{n}\right)\left\|T z_{n}-x^{*}\right\|^{2}-\gamma_{n}\left(1-\gamma_{n}\right)\left\|v_{n}-T z_{n}\right\|^{2} \\
& +2 \beta_{n}\left\langle f\left(z_{n}\right)-v F T z_{n}, x_{n+1}-x^{*}\right\rangle \\
= & \gamma_{n}\left[\sigma_{n}\left\|p_{n}-x^{*}\right\|^{2}+\left(1-\sigma_{n}\right)\left\|T_{n} p_{n}-x^{*}\right\|^{2}-\sigma_{n}\left(1-\sigma_{n}\right)\left\|p_{n}-T_{n} p_{n}\right\|^{2}\right] \\
& +\left(1-\gamma_{n}\right)\left\|T z_{n}-x^{*}\right\|^{2}-\gamma_{n}\left(1-\gamma_{n}\right)\left\|v_{n}-T z_{n}\right\|^{2}+2 \beta_{n}\left\langle f\left(z_{n}\right)-v F T z_{n}, x_{n+1}-x^{*}\right\rangle \\
\leq & \gamma_{n}\left[\left\|p_{n}-x^{*}\right\|^{2}-\sigma_{n}\left(1-\sigma_{n}\right)\left\|p_{n}-T_{n} p_{n}\right\|^{2}\right]+\left(1-\gamma_{n}\right)\left\|z_{n}-x^{*}\right\|^{2} \\
& -\gamma_{n}\left(1-\gamma_{n}\right)\left\|v_{n}-T z_{n}\right\|^{2}+2 \beta_{n}\left\langle f\left(z_{n}\right)-v F T z_{n}, x_{n+1}-x^{*}\right\rangle \\
\leq & \gamma_{n}\left[\left(\left\|x_{n}-x^{*}\right\|+\beta_{n} M_{0}\right)^{2}-\sigma_{n}\left(1-\sigma_{n}\right)\left\|p_{n}-T_{n} p_{n}\right\|^{2}\right] \\
& +\left(1-\gamma_{n}\right)\left(\left\|x_{n}-x^{*}\right\|+\beta_{n} M_{0}\right)^{2}-\gamma_{n}\left(1-\gamma_{n}\right)\left\|v_{n}-T z_{n}\right\|^{2} \\
& +2 \beta_{n}\left\|f\left(z_{n}\right)-v F T z_{n}\right\|\left\|x_{n+1}-x^{*}\right\| \\
\leq & \left(\left\|x_{n}-x^{*}\right\|+\beta_{n} M_{0}\right)^{2}-\gamma_{n} \sigma_{n}\left(1-\sigma_{n}\right)\left\|p_{n}-T_{n} p_{n}\right\|^{2} \\
& -\gamma_{n}\left(1-\gamma_{n}\right)\left\|v_{n}-T z_{n}\right\|^{2}+2 \beta_{n}\left\|f\left(z_{n}\right)-v F T z_{n}\right\|\left\|x_{n+1}-x^{*}\right\|,
\end{aligned}
$$


which immediately yields

$$
\begin{aligned}
& \gamma_{n} \sigma_{n}\left(1-\sigma_{n}\right)\left\|p_{n}-T_{n} p_{n}\right\|^{2}+\gamma_{n}\left(1-\gamma_{n}\right)\left\|v_{n}-T z_{n}\right\|^{2} \\
& \leq\left(\left\|x_{n}-x^{*}\right\|+\beta_{n} M_{0}\right)^{2}-\left\|x_{n+1}-x^{*}\right\|^{2}+2 \beta_{n}\left\|f\left(z_{n}\right)-v F T z_{n}\right\|\left\|x_{n+1}-x^{*}\right\| \\
& =\Gamma_{n}-\Gamma_{n+1}+\beta_{n} M_{0}\left(2\left\|x_{n}-x^{*}\right\|+\beta_{n} M_{0}\right)+2 \beta_{n}\left\|f\left(z_{n}\right)-v F T z_{n}\right\|\left\|x_{n+1}-x^{*}\right\| .
\end{aligned}
$$

Since $\beta_{n} \rightarrow 0, \Gamma_{n}-\Gamma_{n+1} \rightarrow 0,\left\{\gamma_{n}\right\} \subset[a, b] \subset(0,1)$ and $\left\{\sigma_{n}\right\} \subset[c, d] \subset(0,1)$, we have from the boundedness of $\left\{x_{n}\right\}$ and $\left\{z_{n}\right\}$ that

$$
\lim _{n \rightarrow \infty}\left\|p_{n}-T_{n} p_{n}\right\|=\lim _{n \rightarrow \infty}\left\|v_{n}-T z_{n}\right\|=0
$$

It follows that

$$
\left\|v_{n}-T z_{n}\right\|^{2}=\sigma_{n}\left\|p_{n}-T z_{n}\right\|^{2}+\left(1-\sigma_{n}\right)\left\|T_{n} p_{n}-T z_{n}\right\|^{2}-\sigma_{n}\left(1-\sigma_{n}\right)\left\|p_{n}-T_{n} p_{n}\right\|^{2} .
$$

By making use of (3.19) and $\left\{\sigma_{n}\right\} \subset[c, d] \subset(0,1)$, we see that

$$
\lim _{n \rightarrow \infty}\left\|p_{n}-T z_{n}\right\|=\lim _{n \rightarrow \infty}\left\|T_{n} p_{n}-T z_{n}\right\|=0 .
$$

In view of (3.17), (3.18), (3.19) and (3.20), we conclude that $\left\|T z_{n}-x_{n}\right\| \leq\left\|T z_{n}-z_{n}\right\|+\| z_{n}-$ $x_{n}\|\rightarrow 0,\| v_{n}-x_{n}\|\leq\| v_{n}-T z_{n}\|+\| T z_{n}-x_{n} \| \rightarrow 0$,

$$
\begin{aligned}
\left\|z_{n}-x_{n}\right\| & \leq\left\|z_{n}-p_{n}\right\|+\left\|p_{n}-x_{n}\right\| \rightarrow 0, \\
\left\|T z_{n}-z_{n}\right\| & \leq\left\|T z_{n}-p_{n}\right\|+\left\|p_{n}-z_{n}\right\| \rightarrow 0, \\
\left\|y_{n}-x_{n}\right\| & \leq\left\|y_{n}-z_{n}\right\|+\left\|z_{n}-x_{n}\right\| \rightarrow 0,
\end{aligned}
$$

as $n \rightarrow \infty$. Hence,

$$
\begin{aligned}
\left\|x_{n+1}-x_{n}\right\| & =\left\|\beta_{n}\left(f\left(z_{n}\right)-v F T z_{n}\right)+\gamma_{n}\left(v_{n}-x_{n}\right)+\left(1-\gamma_{n}\right)\left(T z_{n}-x_{n}\right)\right\| \\
& \leq \beta_{n}\left\|f\left(z_{n}\right)-v F T z_{n}\right\|+\gamma_{n}\left\|v_{n}-x_{n}\right\|+\left(1-\gamma_{n}\right)\left\|T z_{n}-x_{n}\right\| \\
& \leq \beta_{n}\left\|f\left(z_{n}\right)-v F T z_{n}\right\|+\left\|v_{n}-x_{n}\right\|+\left\|T z_{n}-x_{n}\right\| \rightarrow 0
\end{aligned}
$$

as $n \rightarrow \infty$. Furthermore, from the boundedness of $\left\{x_{n}\right\}$, it follows that there exists a subsequence $\left\{x_{n_{k}}\right\}$ of $\left\{x_{n}\right\}$ such that

$$
\limsup _{n \rightarrow \infty}\left\langle(f-v F) x^{*}, x_{n}-x^{*}\right\rangle=\lim _{k \rightarrow \infty}\left\langle(f-v F) x^{*}, x_{n_{k}}-x^{*}\right\rangle .
$$

Since space $H$ is reflexive and sequence $\left\{x_{n}\right\}$ is bounded, we may assume, without loss of generality, that $x_{n_{k}} \rightarrow \tilde{x}$. Hence, it follows from (3.25) that

$$
\limsup _{n \rightarrow \infty}\left\langle(f-v F) x^{*}, x_{n}-x^{*}\right\rangle=\lim _{k \rightarrow \infty}\left\langle(f-v F) x^{*}, x_{n_{k}}-x^{*}\right\rangle=\left\langle(f-v F) x^{*}, \tilde{x}-x^{*}\right\rangle .
$$

We next claim that $\tilde{x} \in \operatorname{EP}(\Theta)$. In fact, by the definition of $y_{n}$, we get

$$
0 \in \partial_{2}\left(\lambda_{n} \Theta\left(p_{n}, y\right)+\frac{1}{2}\left\|p_{n}-y\right\|^{2}\right)\left(y_{n}\right)+N_{C}\left(y_{n}\right) \text {. }
$$

Hence, there exist $w \in \partial_{2} \Theta\left(p_{n}, \cdot\right)\left(y_{n}\right)$ and $\bar{w} \in N_{C}\left(y_{n}\right)$ such that $\lambda_{n} w+y_{n}-p_{n}+\bar{w}=0$. Thus,

$$
\left\langle y_{n}-p_{n}, y-y_{n}\right\rangle=\lambda_{n}\left\langle w, y_{n}-y\right\rangle+\left\langle\bar{w}, y_{n}-y\right\rangle \text {. }
$$

From $\bar{w} \in N_{C}\left(y_{n}\right)$, we obtain that $\left\langle\bar{w}, y-y_{n}\right\rangle \leq 0 \forall y \in C$. This together with (3.27) implies that $\left\langle y_{n}-p_{n}, y-y_{n}\right\rangle \geq \lambda_{n}\left\langle w, y_{n}-y\right\rangle, \forall y \in C$. From $w \in \partial_{2} \Theta\left(p_{n}, \cdot\right)\left(y_{n}\right)$, we get $\left\langle w, y_{n}-y\right\rangle \geq$ $\Theta\left(p_{n}, y_{n}\right)-\Theta\left(p_{n}, y\right), \forall y \in H$, By the last two inequalities, we have

$$
\lambda_{n}\left(\Theta\left(p_{n}, y\right)-\Theta\left(p_{n}, y_{n}\right)\right) \geq\left\langle p_{n}-y_{n}, y-y_{n}\right\rangle, \quad \forall y \in C .
$$


Owing to (3.18), we have $p_{n_{k}} \rightarrow \tilde{x}$. Also, by (3.23), we get $y_{n_{k}} \rightarrow \tilde{x}$. By the definition of $y_{n}$, we know that $y_{n} \in C, \forall n \geq 1$. Note that subset $C$ is closed and convex set and space $H$ is Hilbert. So, $C$ is weakly closed. Thus, $\tilde{x} \in C$. From $\lambda_{n}<\delta<1$ and (3.28), we infer that

$$
\Theta\left(p_{n_{k}}, y\right)-\Theta\left(p_{n_{k}}, y_{n_{k}}\right)>\left\langle p_{n_{k}}-y_{n_{k}}, y-y_{n_{k}}\right\rangle, \quad \forall y \in C .
$$

Letting $k \rightarrow \infty$, since $\Theta$ is jointly weakly continuous (condition (A3)), we get $\Theta(\tilde{x}, y) \geq 0, \forall y \in C$. This ensures that $\tilde{x} \in \operatorname{EP}(\Theta)$.

We further claim that $\tilde{x} \in \bigcap_{i=0}^{N} \operatorname{Fix}\left(T_{i}\right)$. In fact, we show that $\lim _{n \rightarrow \infty}\left\|p_{n}-T_{l} p_{n}\right\|=0$ for $l=1, \ldots, N$. Note that for $i=1, \ldots, N$,

$$
\begin{aligned}
\left\|p_{n}-T_{n+i} p_{n}\right\| & \leq\left\|p_{n}-p_{n+i}\right\|+\left\|p_{n+i}-T_{n+i} p_{n+i}\right\|+\left\|T_{n+i} p_{n+i}-T_{n+i} p_{n}\right\| \\
& \leq 2\left\|p_{n}-p_{n+i}\right\|+\left\|p_{n+i}-T_{n+i} p_{n+i}\right\| \\
& \leq 2\left(\left\|p_{n}-x_{n}\right\|+\left\|x_{n}-x_{n+i}\right\|+\left\|x_{n+i}-p_{n+i}\right\|\right)+\left\|p_{n+i}-T_{n+i} p_{n+i}\right\| .
\end{aligned}
$$

Hence, from (3.18), (3.19) and (3.24), we get $\lim _{n \rightarrow \infty}\left\|p_{n}-T_{n+i} p_{n}\right\|=0$ for $i=1, \ldots, N$. This immediately implies that

$$
\lim _{n \rightarrow \infty}\left\|p_{n}-T_{l} p_{n}\right\|=0, \quad \text { for } l=1, \ldots, N .
$$

From $p_{n}-x_{n} \rightarrow 0, z_{n}-x_{n} \rightarrow 0$ (due to (3.18), (3.21)) and $x_{n_{k}} \rightarrow \tilde{x}$, we get $p_{n_{k}} \rightarrow \tilde{x}$ and $z_{n_{k}} \rightarrow \tilde{x}$. From (3.22), we have $p_{n_{k}}-T_{l} p_{n_{k}} \rightarrow 0$ for $l=1, \ldots, N$. Note that Lemma 2.3 guarantees the demiclosedness of $I-T_{l}$ at zero for $l=1, \ldots, N$. Thus $\tilde{x} \in \operatorname{Fix}\left(T_{l}\right)$. Since $l$ is an arbitrary element in the finite set $\{1, \ldots, N\}$, we get $\tilde{x} \in \cap_{i=1}^{N} \operatorname{Fix}\left(T_{i}\right)$. Also, note that $\lim _{n \rightarrow \infty}\left\|z_{n}-T z_{n}\right\|=0$ (due to (3.22)). So, utilizing the demiclosedness of $I-T$ at zero, we deduce from $z_{n_{k}} \rightarrow \tilde{x}$ that $\tilde{x} \in \operatorname{Fix}(T)$, which hence yields $\tilde{x} \in \bigcap_{i=0}^{N} \operatorname{Fix}\left(T_{i}\right)$. Consequently, $\tilde{x} \in \bigcap_{i=0}^{N} \operatorname{Fix}\left(T_{i}\right) \cap \operatorname{EP}(\Theta)=\Omega$. In view of (3.26), we get

$$
\limsup _{n \rightarrow \infty}\left\langle\left\langle(f-v F) x^{*}, x_{n}-x^{*}\right\rangle\right\rangle=\left\langle(f-v F) x^{*}, \tilde{x}-x^{*}\right\rangle \leq 0,
$$

which, together with (3.24), leads to

$$
\begin{aligned}
& \limsup _{n \rightarrow \infty}\left\langle(f-v F) x^{*}, x_{n+1}-x^{*}\right\rangle \\
& =\limsup _{n \rightarrow \infty}\left[\left\langle(f-v F) x^{*}, x_{n+1}-x_{n}\right\rangle+\left\langle(f-v F) x^{*}, x_{n}-x^{*}\right\rangle\right] \\
& \leq \limsup _{n \rightarrow \infty}\left[\left\|(f-v F) x^{*}\right\|\left\|x_{n+1}-x_{n}\right\|+\left\langle(f-v F) x^{*}, x_{n}-x^{*}\right\rangle\right] \leq 0 .
\end{aligned}
$$

Note that $\left\{\beta_{n}(\tau-\zeta)\right\} \subset[0,1], \sum_{n=1}^{\infty} \beta_{n}(\tau-\zeta)=\infty$, and

$$
\limsup _{n \rightarrow \infty}\left[\frac{2\left\langle(f-v F) x^{*}, x_{n+1}-x^{*}\right\rangle}{\tau-\zeta}+\frac{3 M}{\tau-\zeta} \cdot \frac{\alpha_{n}}{\beta_{n}}\left\|x_{n}-x_{n-1}\right\|\right] \leq 0 .
$$

Therefore, applying Lemma 2.4 to (3.16), we get $\lim _{k \rightarrow \infty}\left\|x_{n}-x^{*}\right\|=0$.

Case 2. Suppose that there exists $\left\{\Gamma_{n_{k}}\right\} \subset\left\{\Gamma_{n}\right\}$ such that $\Gamma_{n_{k}}<\Gamma_{n_{k}+1}, \forall k \in \mathbb{Z}$, where $\mathbb{Z}$ is the set of all positive integers. Define the mapping $\tau: \mathbb{Z} \rightarrow \mathbb{Z}$ by

$$
\tau(n):=\max \left\{k \leq n: \Gamma_{k}<\Gamma_{k+1}\right\} .
$$

By making use of Lemma 2.5, we get

$$
\Gamma_{\tau(n)} \leq \Gamma_{\tau(n)+1} \quad \text { and } \quad \Gamma_{n} \leq \Gamma_{\tau(n)+1} .
$$


Putting $\Gamma_{n}=\left\|x_{n}-x^{*}\right\|^{2}, \forall n \in \mathbb{Z}$ and using the same inference as in Case 1, we can obtain

$$
\lim _{n \rightarrow \infty}\left\|x_{\tau(n)+1}-x_{\tau(n)}\right\|=0,
$$

and $\lim \sup _{n \rightarrow \infty}\left\langle(f-v F) x^{*}, x_{\tau(n)+1}-x^{*}\right\rangle \leq 0$. In view o $\Gamma_{\tau(n)} \leq \Gamma_{\tau(n)+1}$ and $0<\beta_{\tau(n)}$, we conclude from (3.16) that

$$
\left\|x_{\tau(n)}-x^{*}\right\|^{2} \leq \frac{2}{\tau-\zeta}\left\langle(f-v F) x^{*}, x_{\tau(n)+1}-x^{*}\right\rangle+\frac{3 M}{\tau-\zeta} \cdot \frac{\alpha_{\tau(n)}}{\beta_{\tau(n)}}\left\|x_{\tau(n)}-x_{\tau(n)-1}\right\|,
$$

and hence $\limsup _{n \rightarrow \infty}\left\|x_{\tau(n)}-x^{*}\right\|^{2} \leq 0$. Thus, $\lim _{n \rightarrow \infty}\left\|x_{\tau(n)}-x^{*}\right\|^{2}=0$. From (3.32), we have

$$
\begin{aligned}
& \left\|x_{\tau(n)+1}-x^{*}\right\|^{2}-\left\|x_{\tau(n)}-x^{*}\right\|^{2} \\
& =2\left\langle x_{\tau(n)+1}-x_{\tau(n)}, x_{\tau(n)}-x^{*}\right\rangle+\left\|x_{\tau(n)+1}-x_{\tau(n)}\right\|^{2} \\
& \leq 2\left\|x_{\tau(n)+1}-x_{\tau(n)}\right\|\left\|x_{\tau(n)}-x^{*}\right\|+\left\|x_{\tau(n)+1}-x_{\tau(n)}\right\|^{2} \rightarrow 0 \quad(n \rightarrow \infty) .
\end{aligned}
$$

Thanks to $\Gamma_{n} \leq \Gamma_{\tau(n)+1}$, we get

$$
\begin{aligned}
& \left\|x_{n}-x^{*}\right\|^{2} \leq\left\|x_{\tau(n)+1}-x^{*}\right\|^{2} \\
& \leq\left\|x_{\tau(n)}-x^{*}\right\|^{2}+2\left\|x_{\tau(n)+1}-x_{\tau(n)}\right\|\left\|x_{\tau(n)}-x^{*}\right\|+\left\|x_{\tau(n)+1}-x_{\tau(n)}\right\|^{2} .
\end{aligned}
$$

So it follows from (3.32) that $x_{n} \rightarrow x^{*}$ as $n \rightarrow \infty$. This completes the proof.

Next, we introduce another modified inertial subgradient extragradient algorithm.

Algorithm 3.2. Initial Step: Let $\lambda_{1}>0, \alpha>0, \rho \in(0,1), \delta<\min \left\{1, \frac{1}{2 c_{1}}, \frac{1}{2 c_{2}}\right\}, 0<\mu<\delta$ and $x_{0}, x_{1} \in H$ be arbitrary.

Iterative Steps: Calculate $x_{n+1}$ as follows:

Step 1. Given the iterates $x_{n-1}$ and $x_{n}(n \geq 1)$, choose $\alpha_{n}$ such that $0 \leq \alpha_{n} \leq \bar{\alpha}_{n}$, where

$$
\bar{\alpha}_{n}=\left\{\begin{array}{lc}
\min \left\{\alpha, \frac{\varepsilon_{n}}{\left\|x_{n}-x_{n-1}\right\|}\right\}, & \text { if } x_{n} \neq x_{n-1}, \\
\alpha, & \text { otherwise. }
\end{array}\right.
$$

Step 2. Compute $p_{n}=x_{n}+\alpha_{n}\left(x_{n}-x_{n-1}\right)$ and

$$
y_{n}=\operatorname{argmin}\left\{\lambda_{n} \Theta\left(p_{n}, y\right)+\frac{1}{2}\left\|p_{n}-y\right\|^{2}: y \in C\right\} .
$$

Step 3. Pick $w_{n} \in \partial_{2} \Theta\left(p_{n}, \cdot\right) y_{n}$, construct

$$
C_{n}=\left\{z \in H:\left\langle\left(p_{n}-\lambda_{n} w_{n}\right)-y_{n}, z-y_{n}\right\rangle \leq 0\right\}
$$

(i.e., a half-space whose bounding hyperplane supports $C$ at $y_{n}$ ), and compute

$$
z_{n}=\operatorname{argmin}\left\{\mu \lambda_{n} \Theta\left(y_{n}, y\right)+\frac{1}{2}\left\|p_{n}-y\right\|^{2}: y \in C_{n}\right\} .
$$

Step 4. Calculate $v_{n}=\sigma_{n} p_{n}+\left(1-\sigma_{n}\right) T z_{n}$ and

$$
x_{n+1}=\beta_{n} f\left(z_{n}\right)+\gamma_{n} v_{n}+\left(\left(1-\gamma_{n}\right) I-\beta_{n} v F\right) T_{n} p_{n},
$$

and update

$$
\lambda_{n+1}=\min \left\{\delta, \frac{\mu \Theta\left(y_{n}, z_{n}\right)}{\Theta\left(p_{n}, z_{n}\right)-\Theta\left(p_{n}, y_{n}\right)-c_{1}\left\|p_{n}-y_{n}\right\|^{2}-c_{2}\left\|y_{n}-z_{n}\right\|^{2}+1}\right\} .
$$

Set $n:=n+1$ and return to Step 1 . 
Theorem 3.2. Let $C$ be a nonempty closed convex subset of a real Hilbert space $\mathscr{H}$. Let $\left\{x_{n}\right\}$ be the sequence constructed by Algorithm 3.2. Then $x_{n} \rightarrow x^{*} \in \Omega$, which is a unique solution to the VIP: $\left\langle(v F-f) x^{*}, p-x^{*}\right\rangle \geq 0, \forall p \in \Omega$.

Proof. Utilizing the same inference as in the proof of Theorem 3.2, we deduce that there exists a unique solution $x^{*} \in \Omega=\cap_{i=0}^{N} \operatorname{Fix}\left(T_{i}\right) \cap \operatorname{EP}(\Theta)$ to the $\operatorname{VIP}\left\langle(v F-f) x^{*}, p-x^{*}\right\rangle \geq 0, \forall p \in \Omega$.

Next, we divide the rest of the proof into several steps.

Step 1. We show that inequality (3.4) in the proof of Theorem 3.1 still holds. Indeed, utilizing the same arguments as in the proof of Theorem 3.1, we derive the desired assertion.

Step 2. Show that $\left\{x_{n}\right\}$ is bounded.

Indeed, utilizing the same arguments as in the proof of Theorem 3.1, we deduce that inequalities (3.10)-(3.13) and (3.15) still hold. From $v_{n}=\sigma_{n} p_{n}+\left(1-\sigma_{n}\right) T z_{n}$, we get

$$
\left\|v_{n}-x^{*}\right\| \leq \sigma_{n}\left\|p_{n}-x^{*}\right\|+\left(1-\sigma_{n}\right)\left\|T z_{n}-x^{*}\right\| \leq\left\|p_{n}-x^{*}\right\|
$$

Therefore, Lemma 2.2, (3.13) and (3.15) guarantees that

$$
\begin{aligned}
\left\|x_{n+1}-x^{*}\right\| \leq & \beta_{n}\left(\left\|f\left(z_{n}\right)-f\left(x^{*}\right)\right\|+\left\|f\left(x^{*}\right)-x^{*}\right\|\right)+\gamma_{n}\left\|v_{n}-x^{*}\right\| \\
& +\left(1-\beta_{n}-\gamma_{n}\right)\left\|\left(\frac{1-\gamma_{n}}{1-\beta_{n}-\gamma_{n}} I-\frac{\beta_{n}}{1-\beta_{n}-\gamma_{n}} v F\right) T_{n} p_{n}-x^{*}\right\| \\
\leq & \beta_{n}\left(\zeta\left\|z_{n}-x^{*}\right\|+\left\|f\left(x^{*}\right)-x^{*}\right\|\right)+\gamma_{n}\left\|p_{n}-x^{*}\right\| \\
& +\left(1-\gamma_{n}\right)\left\|\left(I-\frac{\beta_{n}}{1-\gamma_{n}} v F\right) T_{n} p_{n}-\left(I-\frac{\beta_{n}}{1-\gamma_{n}} v F\right) x^{*}+\frac{\beta_{n}}{1-\gamma_{n}}(I-v F) x^{*}\right\| \\
\leq & \beta_{n}\left(\zeta\left\|z_{n}-x^{*}\right\|+\left\|f\left(x^{*}\right)-x^{*}\right\|\right)+\gamma_{n}\left\|p_{n}-x^{*}\right\| \\
& +\left(1-\gamma_{n}-\beta_{n} \tau\right)\left\|p_{n}-x^{*}\right\|+\beta_{n}\left\|(I-v F) x^{*}\right\| \\
\leq & {\left[1-\beta_{n}(\tau-\zeta)\right]\left(\left\|x_{n}-x^{*}\right\|+\beta_{n} M_{0}\right)+\beta_{n}\left(\left\|f\left(x^{*}\right)-x^{*}\right\|+\left\|(I-v F) x^{*}\right\|\right) } \\
\leq & {\left[1-\beta_{n}(\tau-\zeta)\right]\left\|x_{n}-x^{*}\right\|+\beta_{n}(\tau-\zeta) \cdot \frac{M_{0}+\left\|f\left(x^{*}\right)-x^{*}\right\|+\left\|(I-v F) x^{*}\right\|}{\tau-\zeta} } \\
\leq & \max \left\{\left\|x_{n}-x^{*}\right\|, \frac{M_{0}+\left\|f\left(x^{*}\right)-x^{*}\right\|+\left\|(I-v F) x^{*}\right\|}{\tau-\zeta}\right\} .
\end{aligned}
$$

By induction, we obtain

$$
\left\|x_{n}-x^{*}\right\| \leq \max \left\{\left\|x_{1}-x^{*}\right\|, \frac{M_{0}+\left\|f\left(x^{*}\right)-x^{*}\right\|+\left\|(I-v F) x^{*}\right\|}{\tau-\zeta}\right\}, \quad \forall n \geq 1 .
$$

Thus, $\left\{x_{n}\right\}$ is bounded, so are $\left\{p_{n}\right\},\left\{z_{n}\right\},\left\{f\left(z_{n}\right)\right\},\left\{T_{n} p_{n}\right\}$ and $\left\{T z_{n}\right\}$.

Step 3. Show that $\left\{x_{n}\right\}$ converges strongly to $x^{*}$. 
Indeed, utilizing Lemma 2.3, (3.10) and the convexity of the function $h(s)=s^{2} \forall s \in \mathbb{R}$, we obtain that

$$
\begin{aligned}
\| & x_{n+1}-x^{*} \|^{2} \\
\leq & \| \beta_{n}\left(f\left(z_{n}\right)-f\left(x^{*}\right)\right)+\gamma_{n}\left(v_{n}-x^{*}\right)+\left(1-\gamma_{n}\right)\left[\left(I-\frac{\beta_{n}}{1-\gamma_{n}} v F\right) T_{n} p_{n}\right. \\
& \left.-\left(I-\frac{\beta_{n}}{1-\gamma_{n}} v F\right) x^{*}\right] \|^{2}+2 \beta_{n}\left\langle(f-v F) x^{*}, x_{n+1}-x^{*}\right\rangle \\
\leq & \beta_{n} \zeta\left\|z_{n}-x^{*}\right\|^{2}+\gamma_{n}\left(\sigma_{n}\left\|p_{n}-x^{*}\right\|^{2}+\left(1-\sigma_{n}\right)\left\|z_{n}-x^{*}\right\|^{2}\right)+\left(1-\beta_{n} \tau-\gamma_{n}\right)\left\|p_{n}-x^{*}\right\|^{2} \\
& +2 \beta_{n}\left\langle(f-v F) x^{*}, x_{n+1}-x^{*}\right\rangle \\
\leq & {\left[1-\beta_{n}(\tau-\zeta)\right]\left\|p_{n}-x^{*}\right\|^{2}+2 \beta_{n}\left\langle(f-v F) x^{*}, x_{n+1}-x^{*}\right\rangle } \\
\leq & {\left[1-\beta_{n}(\tau-\zeta)\right]\left\{\left\|x_{n}-x^{*}\right\|^{2}+\alpha_{n}\left\|x_{n}-x_{n-1}\right\|\left[2\left\|x_{n}-x^{*}\right\|+\alpha_{n}\left\|x_{n}-x_{n-1}\right\|\right]\right\} } \\
& +2 \beta_{n}\left\langle(f-v F) x^{*}, x_{n+1}-x^{*}\right\rangle \\
\leq & {\left[1-\beta_{n}(\tau-\zeta)\right]\left\|x_{n}-x^{*}\right\|^{2}+\beta_{n}(\tau-\zeta)\left[\frac{2\left\langle(f-v F) x^{*}, x_{n+1}-x^{*}\right\rangle}{\tau-\zeta}+\frac{3 M}{\tau-\zeta} \frac{\alpha_{n}}{\beta_{n}}\left\|x_{n}-x_{n-1}\right\|\right], }
\end{aligned}
$$

where $\sup _{n \geq 1}\left\{\left\|x_{n}-x^{*}\right\|, \alpha_{n}\left\|x_{n}-x_{n-1}\right\|\right\} \leq M$ for some $M>0$. For each $n \geq 1$, we set $\Gamma_{n}=$ $\left\|x_{n}-x^{*}\right\|^{2}, \varepsilon_{n}=\beta_{n}(\tau-\zeta)$ and $\vartheta_{n}=\alpha_{n}\left\|x_{n}-x_{n-1}\right\| 3 M+2 \beta_{n}\left\langle(f-v F) x^{*}, x_{n+1}-x^{*}\right\rangle$. Then (3.33) can be rewritten as $\Gamma_{n+1} \leq\left(1-\varepsilon_{n}\right) \Gamma_{n}+\vartheta_{n}$.

Finally, we show the convergence of $\left\{\Gamma_{n}\right\}$ to zero by the following two cases:

Case 1. Suppose that there exists a positive integer $n_{0}$ such that $\left\{\Gamma_{n}\right\}$ is non-increasing for $n \geq n_{0}$. Then we obtain that the $\operatorname{limit}_{n \rightarrow \infty}\left\|x_{n}-x^{*}\right\|$ exists and $\Gamma_{n}-\Gamma_{n+1} \rightarrow 0$ as $n \rightarrow \infty$. So, it follows from (3.4), (3.15) and (3.33) that

$$
\begin{aligned}
& \left\|x_{n+1}-x^{*}\right\|^{2} \\
& \leq\left[\beta_{n} \zeta+\gamma_{n}\left(1-\sigma_{n}\right)\right]\left\|z_{n}-x^{*}\right\|^{2}+\left[1-\beta_{n} \tau-\gamma_{n}\left(1-\sigma_{n}\right)\right]\left\|p_{n}-x^{*}\right\|^{2} \\
& \quad+2 \beta_{n}\left\langle(f-v F) x^{*}, x_{n+1}-x^{*}\right\rangle \\
& \leq\left[1-\beta_{n}(\tau-\zeta)\right]\left\|p_{n}-x^{*}\right\|^{2}-\left[\beta_{n} \zeta+\gamma_{n}\left(1-\sigma_{n}\right)\right]\left[\left(1-\lambda_{n+1}\right)\left\|p_{n}-z_{n}\right\|^{2}\right. \\
& \left.\quad+\lambda_{n+1}\left(1-2 \lambda_{n} c_{1}\right)\left\|p_{n}-y_{n}\right\|^{2}+\lambda_{n+1}\left(1-2 \lambda_{n} c_{2}\right)\left\|z_{n}-y_{n}\right\|^{2}\right]+\beta_{n} M_{1} \\
& \leq\left\|x_{n}-x^{*}\right\|^{2}+\alpha_{n}\left\|x_{n}-x_{n-1}\right\| 3 M-\left[\beta_{n} \zeta+\gamma_{n}\left(1-\sigma_{n}\right)\right]\left[\left(1-\lambda_{n+1}\right)\left\|p_{n}-z_{n}\right\|^{2}\right. \\
& \left.\quad+\lambda_{n+1}\left(1-2 \lambda_{n} c_{1}\right)\left\|p_{n}-y_{n}\right\|^{2}+\lambda_{n+1}\left(1-2 \lambda_{n} c_{2}\right)\left\|z_{n}-y_{n}\right\|^{2}\right]+\beta_{n} M_{1},
\end{aligned}
$$

where $\sup _{n \geq 1} 2\left\|(f-v F) x^{*}\right\|\left\|x_{n}-x^{*}\right\| \leq M_{1}$ for some $M_{1}>0$. This immediately yields

$$
\begin{aligned}
& {\left[\beta_{n} \zeta+\gamma_{n}\left(1-\sigma_{n}\right)\right]\left[\left(1-\lambda_{n+1}\right)\left\|p_{n}-z_{n}\right\|^{2}+\lambda_{n+1}\left(1-2 \lambda_{n} c_{1}\right)\left\|p_{n}-y_{n}\right\|^{2}\right.} \\
& \left.\quad+\lambda_{n+1}\left(1-2 \lambda_{n} c_{2}\right)\left\|z_{n}-y_{n}\right\|^{2}\right] \leq \Gamma_{n}-\Gamma_{n+1}+\alpha_{n}\left\|x_{n}-x_{n-1}\right\| 3 M+\beta_{n} M_{1}
\end{aligned}
$$

Since $\Gamma_{n}-\Gamma_{n+1} \rightarrow 0, \beta_{n} \rightarrow 0, \alpha_{n}\left\|x_{n}-x_{n-1}\right\| \rightarrow 0$ and $\liminf _{n \rightarrow \infty} \gamma_{n}\left(1-\sigma_{n}\right)>0$, we obtain from $\rho \mu<\lambda_{n}<\delta$ that

$$
\lim _{n \rightarrow \infty}\left\|p_{n}-z_{n}\right\|=\lim _{n \rightarrow \infty}\left\|p_{n}-y_{n}\right\|=\lim _{n \rightarrow \infty}\left\|z_{n}-y_{n}\right\|=0
$$


In view of $p_{n}=x_{n}+\alpha_{n}\left(x_{n}-x_{n-1}\right)$, we get $\lim _{n \rightarrow \infty}\left\|p_{n}-x_{n}\right\|=0$. It follows from (3.13) that

$$
\begin{aligned}
\left\|x_{n+1}-x^{*}\right\|^{2} \leq & \gamma_{n}\left\|v_{n}-x^{*}\right\|^{2}+\left(1-\gamma_{n}\right)\left\|T_{n} p_{n}-x^{*}\right\|^{2}-\gamma_{n}\left(1-\gamma_{n}\right)\left\|v_{n}-T_{n} p_{n}\right\|^{2} \\
& +2 \beta_{n}\left\langle f\left(z_{n}\right)-v F T_{n} p_{n}, x_{n+1}-x^{*}\right\rangle \\
\leq & \gamma_{n}\left[\left\|p_{n}-x^{*}\right\|^{2}-\sigma_{n}\left(1-\sigma_{n}\right)\left\|p_{n}-T z_{n}\right\|^{2}\right]+\left(1-\gamma_{n}\right)\left\|p_{n}-x^{*}\right\|^{2} \\
& -\gamma_{n}\left(1-\gamma_{n}\right)\left\|v_{n}-T_{n} p_{n}\right\|^{2}+2 \beta_{n}\left\langle f\left(z_{n}\right)-v F T_{n} p_{n}, x_{n+1}-x^{*}\right\rangle \\
\leq & \left(\left\|x_{n}-x^{*}\right\|+\beta_{n} M_{0}\right)^{2}-\gamma_{n} \sigma_{n}\left(1-\sigma_{n}\right)\left\|p_{n}-T z_{n}\right\|^{2} \\
& -\gamma_{n}\left(1-\gamma_{n}\right)\left\|v_{n}-T_{n} p_{n}\right\|^{2}+2 \beta_{n}\left\|f\left(z_{n}\right)-v F T_{n} p_{n}\right\|\left\|x_{n+1}-x^{*}\right\|,
\end{aligned}
$$

which immediately yields

$$
\begin{aligned}
& \gamma_{n} \sigma_{n}\left(1-\sigma_{n}\right)\left\|p_{n}-T z_{n}\right\|^{2}+\gamma_{n}\left(1-\gamma_{n}\right)\left\|v_{n}-T_{n} p_{n}\right\|^{2} \\
& \leq \Gamma_{n}-\Gamma_{n+1}+\beta_{n} M_{0}\left(2\left\|x_{n}-x^{*}\right\|+\beta_{n} M_{0}\right)+2 \beta_{n}\left\|f\left(z_{n}\right)-v F T_{n} p_{n}\right\|\left\|x_{n+1}-x^{*}\right\| .
\end{aligned}
$$

Since $\beta_{n} \rightarrow 0, \Gamma_{n}-\Gamma_{n+1} \rightarrow 0,\left\{\gamma_{n}\right\} \subset[a, b] \subset(0,1)$ and $\left\{\sigma_{n}\right\} \subset[c, d] \subset(0,1)$, we have from the boundedness of $\left\{x_{n}\right\},\left\{z_{n}\right\}$ and $\left\{T_{n} p_{n}\right\}$ that

$$
\lim _{n \rightarrow \infty}\left\|p_{n}-T z_{n}\right\|=\lim _{n \rightarrow \infty}\left\|v_{n}-T_{n} p_{n}\right\|=0 .
$$

Note that

$$
\left\|v_{n}-T_{n} p_{n}\right\|^{2} \sigma_{n}\left\|p_{n}-T_{n} p_{n}\right\|^{2}+\left(1-\sigma_{n}\right)\left\|T z_{n}-T_{n} p_{n}\right\|^{2}-\sigma_{n}\left(1-\sigma_{n}\right)\left\|p_{n}-T z_{n}\right\|^{2} .
$$

So it follows from (3.34) and $\left\{\sigma_{n}\right\} \subset[c, d] \subset(0,1)$ that

$$
\lim _{n \rightarrow \infty}\left\|p_{n}-T_{n} p_{n}\right\|=\lim _{n \rightarrow \infty}\left\|T z_{n}-T_{n} p_{n}\right\|=0 .
$$

Thus, from (3.34) and (3.35), we deduce that $\left\|v_{n}-x_{n}\right\| \leq\left\|v_{n}-T_{n} p_{n}\right\|+\left\|T_{n} p_{n}-p_{n}\right\|+\| p_{n}-$ $x_{n}\|\rightarrow 0,\| T_{n} p_{n}-x_{n}\|\leq\| T_{n} p_{n}-v_{n}\|+\| v_{n}-x_{n} \| \rightarrow 0$ as $n \rightarrow \infty$. Hence,

$$
\begin{aligned}
\left\|x_{n+1}-x_{n}\right\| & =\left\|\beta_{n}\left(f\left(z_{n}\right)-v F T_{n} p_{n}\right)+\gamma_{n}\left(v_{n}-x_{n}\right)+\left(1-\gamma_{n}\right)\left(T_{n} p_{n}-x_{n}\right)\right\| \\
& \leq \beta_{n}\left\|f\left(z_{n}\right)-v F T_{n} p_{n}\right\|+\left\|v_{n}-x_{n}\right\|+\left\|T_{n} p_{n}-x_{n}\right\| \rightarrow 0
\end{aligned}
$$

as $n \rightarrow \infty$. In addition, utilizing the similar arguments to those of (3.30) and (3.31), we have $\limsup _{n \rightarrow \infty}\left\langle(f-v F) x^{*}, x_{n}-x^{*}\right\rangle \leq 0$, and $\limsup _{n \rightarrow \infty}\left\langle(f-v F) x^{*}, x_{n+1}-x^{*}\right\rangle \leq 0$. Note that $\left\{\beta_{n}(\tau-\zeta)\right\} \subset[0,1], \sum_{n=1}^{\infty} \beta_{n}(\tau-\zeta)=\infty$, and

$$
\limsup _{n \rightarrow \infty}\left[\frac{2\left\langle(f-v F) x^{*}, x_{n+1}-x^{*}\right\rangle}{\tau-\zeta}+\frac{3 M}{\tau-\zeta} \cdot \frac{\alpha_{n}}{\beta_{n}}\left\|x_{n}-x_{n-1}\right\|\right] \leq 0 .
$$

Therefore, using Lemma 2.4, we have $\lim _{n \rightarrow 0}\left\|x_{n}-x^{*}\right\|=0$.

Case 2. Suppose that there exists $\left\{\Gamma_{n_{k}}\right\} \subset\left\{\Gamma_{n}\right\}$ such that $\Gamma_{n_{k}}<\Gamma_{n_{k}+1}, \forall k \in \mathbb{Z}$. Define the mapping $\tau: \mathbb{Z} \rightarrow \mathbb{Z}$ by $\tau(n):=\max \left\{k \leq n: \Gamma_{k}<\Gamma_{k+1}\right\}$. In the remainder of the proof, utilizing the same arguments as in Case 2 of the proof of Theorem 3.1, we obtain the desired assertion. This completes the proof.

\section{Acknowledgments}

The author thanks the anonymous referees for useful suggestions, which greatly improved the presentation of this paper. 


\section{REFERENCES}

[1] Y. Shehu, J.C. Yao, Rate of convergence for inertial iterative method for countable family of certain quasinonexpansive mappings, J. Nonlinear Convex Anal. 21 (2020), 533-541.

[2] L.C. Ceng, C.T. Pang, C.F. Wen, Multi-step extragradient method with regularization for triple hierarchical variational inequalities with variational inclusion and split feasibility constraints, J. Inequal. Appl. 2014 (2014), Article ID 492.

[3] L.C. Ceng, C.F. Wen, Systems of variational inequalities with hierarchical variational inequality constraints for asymptotically nonexpansive and pseudocontractive mappings, Rev. R. Acad. Cienc. Exactas Fis. Nat. Ser. A Mat. RACSAM 113 (2019), 2431-2447.

[4] S.Y. Cho, X. Qin, L. Wang, Strong convergence of a splitting algorithm for treating monotone operators, Fixed Point Theory Appl. 2014 (2014), Article ID 94.

[5] S.Y. Cho, et al., Viscosity approximation splitting methods for monotone and nonexpansive operators in Hilbert spaces, J. Nonlinear Convex Anal. 19 (2018), 251-264.

[6] N.T. An, N.M. Nam, X. Qin, Solving k-center problems involving sets based on optimization techniques, J. Global Optim. 76 (2020), 189-209.

[7] T.H. Cuong, J.C. Yao, N.D. Yen, Qualitative properties of the minimum sum-of-squares clustering problem, Optimization, 69 (2020), 2131-2154.

[8] D.R. Sahu, J.C. Yao, M. Verma, K.K. Shukla, Convergence rate analysis of proximal gradient methods with applications to composite minimization problems, Optimization, 70 (2021), 75-100.

[9] P. Cubiotti, J.C. Yao, On the Cauchy problem for a class of differential inclusions with applications, Appl. Anal. 99 (2020), 2543-2554.

[10] L.V. Nguyen, Q.H. Ansari, X. Qin, Weak sharpness and finite convergence for solutions of nonsmooth variational inequalities in Hilbert spaces, Appl. Math. Optim. (2020), doi: 10.1007/s00245-020-09662-7.

[11] G.M. Korpelevich, The extragradient method for finding saddle points and other problems, Ekonomikai Matematicheskie Metody 12 (1976) 747-756.

[12] L.C. Ceng, Y.C. Liou, C.F. Wen, Y.J. Wu, Hybrid extragradient viscosity method for general system of variational inequalities, J. Inequal. Appl. 2015 (2015), 150.

[13] L.C. Ceng, Q.H. Ansari, S. Schaible, Hybrid extragradient-like methods for generalized mixed equilibrium problems, systems of generalized equilibrium problems and optimization problems, J. Global Optim. 53 (2012) 69-96.

[14] L.C. Ceng, N. Hadjisavvas, N.C. Wong, Strong convergence theorem by a hybrid extragradient-like approximation method for variational inequalities and fixed point problems, J. Global Optim. 46 (2010) 635-646.

[15] L.V. Nguyen, X. Qin, Some results on strongly pseudomonotone quasi-variational inequalities, Set-Valued Var. Anal. 28 (2020), 239-257.

[16] Y. Shehu, X. Qin, J.C. Yao, Weak and linear convergence of proximal point algorithm with reflections, J. Nonlinear Convex Anal. 22 (2021), 299-307.

[17] Y. Censor, A. Gibali, S. Reich, The subgradient extragradient method for solving varia- tional inequalities in Hilbert space, J. Optim. Theory Appl. 148 (2011) 318-335.

[18] X. Qin, S.Y. Cho, L. Wang, Iterative algorithms with errors for zero points of m-accretive operators, Fixed Point Theory Appl. 2013 (2013), 148.

[19] B. Tan, S. Xu, S. Li, Inertial shrinking projection algorithms for solving hierarchical variational inequality problems, J. Nonlinear Convex Anal. 21 (2020), 871-884.

[20] J. Fan, L. Liu, X. Qin, A subgradient extragradient algorithm with inertial effects for solving strongly pseudomonotone variational inequalities, Optimization, 69 (2020), 2199-2215.

[21] S.Y. Cho, Strong convergence analysis of a hybrid algorithm for nonlinear operators in a Banach space, J. Appl. Anal. Comput. 8 (2018), 19-31.

[22] X. Qin, L. Wang, J.C. Yao, Inertial splitting method for maximal monotone mappings, J. Nonlinear Convex Anal. 21 (2020), 2325-2333.

[23] D.V. Thong, D.V. Hieu, Modified subgradient extragradient method for variational in- equality problems, Numer. Algorithms 79 (2018) 597-610. 
[24] D.V. Thong, D.V. Hieu, Inertial subgradient extragradient algorithms with line-search process for solving variational inequality problems and fixed point problems, Numer. Algorithms 80 (2019) 1283-1307.

[25] S.Y. Cho, A monotone Bregan projection algorithm for fixed point and equilibrium problems in a reflexive Banach space, Filomat, 34 (2020), 1487-1497.

[26] V. Dadashi, O.S. Iyiola, Y. Shehu, The subgradient extragradient method for pseudomonotone equilibrium problems, Optimization 69 (2020), 901-923.

[27] S.Y. Cho, Generalized mixed equilibrium and fixed point problems in a Banach space, J. Nonlinear Sci. Appl. 9 (2016), 1083-1092.

[28] L.C. Ceng, Y.C. Lin, C.F. Wen, Iterative methods for triple hierarchical variational inequalities with mixed equilibrium problems, variational inclusions, and variational inequalities constraints, J. Inequal. Appl. 2015 (2015), 16.

[29] S.Y. Cho, A convergence theorem for generalized mixed equilibrium problems and multivalued asymptotically nonexpansive mappings, J. Nonlinear Convex Anal. 21 (2020), 1017-1026.

[30] W. Cottle, J.C. Yao, Pseudo-monotone complementarity problems in Hilbert space, J. Optim. Theory Appl. 75 (1992), 281-295.

[31] H.K. Xu, T.H. Kim, Convergence of hybrid steepest-descent methods for variational in- equalities, J. Optim. Theory Appl. 119 (2003), 185-201.

[32] K. Goebel, S. Reich, Uniform Convexity, Hyperbolic Geometry, and Nonexpansive Mappings, Marcel Dekker, New York, 1984.

[33] P.E. Maingé, Strong convergence of projected subgradient methods for nonsmooth and nonstrictly convex minimization, Set-Valued Anal. 16 (2008), 899-912. 